\title{
Propiedades Psicométricas de una Prueba de Recuerdos Flashbulb
}

\author{
Psychometric Properties of a Flashbulb Memories Test
}

William Tamayo-Agudelo ${ }^{1}$, Liliana Chaves Castãno ${ }^{2}$ y Julián Cárdenas ${ }^{3}$

\section{Resumen}

Los Recuerdos Flashbulb (MFB) son recuerdos autobiográficos asociados con el momento de recepción de una noticia impactante. El presente estudio buscó determinar las propiedades psicométricas de una prueba que mide estos recuerdos y los factores determinantes para su formación. Los cuestionarios de FBM deben adaptarse rápidamente para que sean utilizados poco tiempo después de que algún acontecimiento inesperado ocurre. Participaron 380 adultos colombianos de ambos sexos. Se describen los criterios seguidos para la calificación de las respuestas. Los análisis factoriales exploratorios se realizaron sobre matrices de correlaciones policóricas, confirmándose la unidimensionalidad de cada una de las partes de la prueba. Se estimaron los coeficientes de confiabilidad, juzgados como aceptables, aunque luego de calcular los intervalos de confianza para los alfas de Cronbach, se encontraron límites inferiores bajos en dos dimensiones. Se considera que una prueba de este tipo puede ser útil en la investigación de recuerdos compartidos por las comunidades.

Palabras clave: recuerdos flashbulb, muerte de Osama bin Laden, análisis factorial

\begin{abstract}
Flashbulb memories are autobiographical memories of specific moments of high emotional impact. This study tested the psychometric properties of a test that aims to measure flashbulb memories and factors associated with their formation. Flashbulb memory questionnaires need to be adapt rapidly to be useful shortly after the index event has occurred. The study involved 380 adults of both sexes. Inclusion and exclusion criteria and fully described. Exploratory factor analysis were conducted using polychoric correlations, confirming the unidimensionality of each part of the test. Reliability coefficients were calculated and fell within the range for acceptable reliability although the lower limits of the confidence intervals were low for two dimensions of the test. Overall, we consider a test of this type to be useful in the investigation of shared memories in the community.
\end{abstract}

Keywords: Flashbulb memories, Osama bin Laden, factor analysis

\footnotetext{
${ }^{1}$ Profesor Instructor, Universidad Cooperativa de Colombia-Medellín. Carrera 47 Número 37 Sur 18, Envigado, Antioquia, Colombia. Tel.: (57+4) 4446065 ext. 4156. Correo: william.tamayoa@ campusucc.edu.co

${ }^{2}$ Vicedecana Facultad de Ciencias Sociales Humanas, Universidad de Antioquia, Colombia. Calle 70 No. 52 - 21 Apartado Aéreo 1226, Colombia. Tel.: (57+4) 2198332. Correo: Liliana.chaves@udea.edu.co

${ }^{3}$ Profesor de tiempo completo, Departamento de Sociología, Universidad de Antioquia, Colombia. Calle 70 No. 52 - 21

- Apartado Aéreo 1226, Colombia. Tel.: (57+4) 2198332. Correo: julian.cardenash@gmail.com
} 


\section{Introducción}

Múltiples son las consecuencias personales y sociales que se desencadenan luego de que se es testigo de un hecho inesperado o se recibe una noticia impactante que afecta a una comunidad en general. Una consecuencia psicológica básica es que se desencadenan respuestas emocionales y cognitivas que se articulan para evaluar el acontecimiento y dar una respuesta conductual. Dicha articulación, sin embargo, puede dar lugar también a la formación de recuerdos personales relacionados con el momento en que se recibió o se vivió el hecho, recuerdos que en sí mismos parecen irrelevantes: personas con quienes se encontraba, fuente de la información, momento del día, lugar, reacciones y emociones experimentadas. Este tipo de recuerdos episódicos se caracterizan fenomenológicamente por ser vívidos y se conocen como Recuerdos Flashbulb o Recuerdos Destello (MFB).

Los instrumentos para medir las variables implicadas en la formación y mantenimiento de los MFB deben elaborarse rápidamente después de acontecido el evento que se desea investigar. La literatura en este campo de investigación ha determinado las principales variables y sus principales categorías. El reto en cada investigación es adecuar de una manera satisfactoria los ítems que corresponden a variables cuya definición responde a los acontecimientos cambiantes de cada evento.

Brown y Kulik (1977) elaboraron por primera vez un cuestionario para medir los MFB. Partiendo de la pregunta ¿Recuerda las circunstancias en las que escuchó por primera vez $q u e . . . ?$, estos autores indagaron por los recuerdos del contexto de recepción de nueve noticias tales como el asesinato del presidente J. F. Kennedy, Martin Luther King, Malcom X, el intento de asesinato de Ronald Regan o la muerte de Francisco Franco en España. Brown y Kulik (1977) al analizar las respuestas de los informantes, descubrieron que al menos el $50 \%$ de los participantes coincidían en seis tipos de información: lugar en donde estaban cuando escucharon la noticia, actividad que estaban realizando, informante, afecto manifestado por las personas que los acompañaban, afecto propio y consecuencias que tuvo el evento. Además, en una escala de 1 a 5 , los autores pidieron a los participantes que evaluaran las consecuencias de estos hechos con respecto a sus vidas y por último, para establecer el papel que juega la repetición como determinante en la formación de un MFB, los informantes promediaron qué tantas veces habían hablado de los eventos.

Este esquema inicial de cuestionario, con algunas variaciones, ha permanecido hasta hoy como base de los estudios en MFB. Conway et al., en 1994, por ejemplo, utilizaron la misma pregunta inicial para diferenciar sujetos que recordasen las circunstancias que rodearon la recepción de la noticia de la renuncia de Margaret Thatcher como primera ministra de Inglaterra. En ese cuestionario, Conway et al. (1994) diferenciaron los atributos de la MFB de los mecanismos de codificación y de mantenimiento.

Para medir los mecanismos de codificación, los autores preguntaron por los niveles de sorpresa, la intensidad de esta, la valencia y el nombre de la emoción experimentada. La importancia personal y nacional atribuida a la renuncia de Margaret Thatcher fue otra de las variables tomadas en cuenta junto con los conocimientos previos acerca del periodo de gobierno de la Primera Ministra, el interés prestado a los asuntos políticos y otros recuerdos relacionados. En cuanto a los mecanismos de mantenimiento de las MFB, Conway et al. (1994), interrogaron a los participantes por la frecuencia con que se habló, pensóyse buscó información relacionada con el hecho en los medios de comunicación. Estos autores no entregaron un reporte de los datos de confiabilidad o validez de dicho cuestionario. Sin embargo, el modelo de ecuación estructural para explicar la formación de las MFB que Conway et al. (1994) propusieron sirviéndose de este instrumento parece una prueba fuerte de sus propiedades para medir las variables latentes predichas por la teoría.

Un tercer cuestionario compuesto de ocho apartados que intentan medir los factores involucrados en la formación y mantenimiento de las MFB de la muerte del rey Baudouin de Bélgica acontecida en 1993, fue elaborado por Finkenaueret et al. (1998). El primer apartado evalúa cinco categorías canónicas de las MFB: día y hora exacta en que se enteró, lugar donde se encontraba, personas que estaban presentes, 
actividad que estaba realizando y hasta cinco detalles específicos del momento en que recibió la noticia. El segundo apartado evalúa los recuerdos del acontecimiento como tal, es decir, la memoria del evento. Los participantes responden preguntas tales como el día, la fecha y la hora de la muerte, el lugar y la causa de la muerte del rey Baudouin y quién fue la primera persona que dio las condolencias a la reina de Bélgica. El tercer apartado de este cuestionario mide la repetición abierta (over rehearsal) de la noticia a través de tres ítems que indagan por el seguimiento del evento en los medios, las conversaciones generales acerca del mismo y las conversaciones centradas en detalles específicos de los acontecimientos.

Los estados emocionales se evalúan en este cuestionario a través de 3 ítems en escalas de 1 a 7. Los participantes evaluaban qué tanto los alteró la noticia, qué tanto los impactó emocionalmente y qué tan conmovidos se sintieron. Para medir la sorpresa, los participantes debían evaluar en una escala de 1 a 7 , qué tanta sorpresa o asombro despertó en ellos la noticia. En cuanto a la evaluación de la novedad, en dos escalas de 1 a 7 , los entrevistados consideraban que tan usual o inusual fue para ellos la muerte del rey y si consideraban que el evento fue común o poco común. Por último, este cuestionario evalúa la importancia y las consecuencias personales atribuidas al evento y las actitudes de los participantes hacia la familia real en términos de simpatía o antipatía.

Esta prueba es mucho más completa que las ideadas por Brown y Kulik (1977) y Conway et al. (1994) y abarca las diversas variables involucradas en la formación y mantenimiento de las MFB. En cuanto a las bases teóricas que fundamentan este cuestionario, los autores acentúan los desarrollos en la investigación psicológica en torno a la evaluación cognitiva de las emociones (Moreira \& Gamboa, 2016) y las consecuencias que dicha evaluación tendría en la formulación de un modelo de formación de las MFB. De nuevo, los autores no entregan un análisis detallado de las propiedades del instrumento, aunque cabe aclarar que las variables observables (aquellas que recoge la prueba) son la base para realizar los modelos de ecuación estructural que permiten finalmente a Finkenauer et al. (1998) concluir que su modelo alcanza índices más aceptables para la explicación de la formación y mantenimiento de la MFB de la muerte del rey Baudouin comparado con los de Brown y Kulik (1977) y Conway et al. (1994).

La prueba creada por Finkenauer et al. (1998) es retomada y modificada por Er (2003) para postular un nuevo modelo de formación para las MFB basado en los recuerdos del contexto de recepción de la noticia del terremoto de Mármaraque afectó a Estambul, Kocaeli y otras regiones de Turquía en 1999. Luego de un análisis factorial exploratorio, el instrumento quedó conformado por 32 ítems. Er (2003) no describe los ítems eliminados ni la conformación final de los factores. Por otra parte, a pesar de que menciona que examinó la consistencia interna de cada sub dimensión, dichos datos no aparecen en el artículo. No obstante, el análisis factorial confirmatorio realizado a través de los modelos de ecuación estructural, arrojan índices satisfactorios (Er, 2003).

Como puede inferirse de los apartados anteriores, el estudio moderno de las MFB se basa en la conjunción de teorías cognitivas y sociales que se ven reflejadas en los instrumentos utilizados. Cada una de las variables implicadas en la formación y mantenimiento de una MFB está representada por un conjunto de ítems que en sí mismo puede ser considerado como un test independiente. $\mathrm{Y}$ esto tiene sentido si se observa el desarrollo de la teoría acerca de estos recuerdos.

Brown y Kulik (1977) enfatizaron, sin desarrollarlo teóricamente, en variables emocionales y cognitivas como la sorpresa y la importancia/consecuencialidad atribuida al evento, para entender la formación de una MFB. Para ello, se apoyaron principalmente en la hipótesis de un mecanismo neurofisiológico diferenciado (el nowprint! de Livingstone, 1967) que podía explicar la aparente vividez de las MFB.

Posteriormente, Conway et al. (1994) agregaron una variable cognitiva organizadora (el conocimiento previo) como factor de codificación y organización de la información y la variable repetición como factor de mantenimiento. Estas dos variables no habían sido tomadas en cuenta en el estudio original de Brown y Kulik (1977), así que tuvieron que ser agregadas independientemente 
en el cuestionario creado por Conway et al. (1994).

El gran aporte de Finkenauer et al. (1998) a los estudios de MFB fue la refinación de la teoría de las emociones integrada a estudios de memoria de este tipo. Finkenauer et al. (1998) retoman los avances investigativos en materia de emocionalidad. En efecto, los avances investigativos en el área de las emociones les permite afirmar que la conceptualización de la emoción no puede reducirse a un simple estado de activación subjetivo. Por el contrario, la aproximación cognitiva a las emociones había entregado bastantes evidencias acerca del papel central que juega la evaluación cognitiva inicial en la determinación de las mismas (Fridja, Kuipers, \& ter Schure, 1989) y esta evaluación a su vez se ve afectada por otras variables como las actitudes, los conocimientos previos y las expectativas de los sujetos. Queda claro, entonces, que la reconceptualización de la emocionalidad realizada por Finkenauer et al. (1998) permitió el fortalecimiento de la teoría de las MFB y vino a agregarse como un factor independiente más que tomar en cuenta los instrumentos de evaluación.

Por tanto, el objetivo principal de este estudio fue determinar las propiedades psicométricas de un instrumento de MFB para un acontecimiento internacional en población colombiana. Para la presente investigación, se retomó el cuestionario descrito por Luminet y Curci (2009) compuesto por siete dimensiones teóricamente reconocidas como fundamentales para la formación de un MFB. Sin embargo, este cuestionario fue desarrollado para evaluar la memoria del contexto de recepción de la noticia de los atentados a las Torres Gemelas de Nueva York, más conocido como 11-S (Luminet et al., 2004). Por esa razón, buena parte del cuestionario hubo de adaptarse a las características de un acontecimiento diferente: la noticia de la muerte de Osama bin Laden en un operativo realizado por un cuerpo élite del ejército norteamericano.

\section{Metodología}

De acuerdo con la clasificación de Montero y León (2007), se puede considerar como un estudio instrumental.

\section{Participantes}

A través de la técnica de muestreo no probabilística bola de nieve, se reclutaron 380 habitantes de la ciudad de Medellín con un rango de edad entre 20 y 89 años $\left(M_{\text {edad }}=37 ; D E=13,4\right)$. Participaron 171 hombres (45\%) y 209 mujeres (55\%) que respondieron la prueba de manera individual luego de firmar el consentimiento informado. $\mathrm{Si}$ el consentimiento informado carecía de firma, número de identificación, hora y fecha de diligenciamiento, era descartado de inmediato. Debido a esto no se tomaron en cuenta 55 instrumentos diligenciados. Las características sociodemográficas de los participantes pueden encontrarse en la Tabla 1.

Tabla 1. Características sociodemográficas de la muestra

\begin{tabular}{lc}
\hline \multicolumn{1}{c}{ Estado civil } & $\%$ \\
\hline Soltero & 44.7 \\
Casado & 26.8 \\
Unión libre & 15.8 \\
Separado & 7.4 \\
Viudo & 3.9 \\
Otro & 0.5 \\
No responde & 0.8 \\
$\quad$ Nivel de estudios & \\
Sin estudios & 0.5 \\
Primaria incompleta & 4.5 \\
Primaria completa & 4.7 \\
Secundaria incompleta & 9.5 \\
Secundaria completa & 26.3 \\
Técnico/Tecnológico & 27.9 \\
Universitario & 20.3 \\
Posgrado & 5.5 \\
No responde & 0.8 \\
\hline
\end{tabular}

\section{Instrumento}

La prueba utilizada originalmente fue desarrollada en el marco de un proyecto trasnacional acerca de los MFB de los ataques a las Torres Gemelas en el año 2001 (Curci \& Luminet, 2006). Posteriormente, se reportó en diversas investigaciones (Luminet \& Curci, 2009; Luminet, et al., 2004). Para este estudio, se retomó el modelo descrito por Luminet y Curci (2009). A continuación se detallan las subescalas que representan las dimensiones relacionadas con la formación de los MFB.

\section{Recuerdos Flashbulb o Memoria del contexto} de recepción (MFB). Siguiendo las categorías canónicas, los participantes reportaron sus 
recuerdos acerca de (a) la hora en la que recibieron la noticia, (b) lugar en el cual se encontraban cuando recibieron la noticia, (c) fuente de la información, (d) personas que se encontraban presentes en el momento de la recepción de la noticia, (e) actividad que se realizaba justo en ese momento. Las respuestas fueron entregadas de manera abierta. La calificación fue 0 si no dio ninguna respuesta y 1 si el participante entregaba una. El puntaje máximo alcanzado en este apartado fue de 5 . En el caso del ítem $a$, se puntuó con 0 toda respuesta que no indicaba una hora específica (v.g., en la mañana, en la tarde o en la noche). En el ítem e,respuestas que no indicaban una actividad específica también recibieron 0 como calificación.

Para evitar un efecto techo en los análisis, se siguió el método introducido por Finkenauer et al. (1998) que consiste en que los participantes deben recordar hasta cinco (5) detalles específicos que acompañaron la recepción de la noticia. Los detalles fueron calificados siguiendo cuatro criterios básicos, dos de ellos propuestos por Finkenauer et al. (1998), y otros dos adicionales: (1) el detalle responde a un aspecto cambiante del ambiente que difícilmente puede ser inferido por la familiaridad del sujeto con el espacio (v.g., el perro estaba en frente echado sobre una poltrona= 1; mi mamá me traía un jugo= 1); (2) el recuerdo corresponde a la posición espacial del participante en relación al ambiente en ese momento específico (estaba sentada en la cabecera de la mesa del comedor=1); (3) el detalle responde a aspectos del entorno o personales que han sido considerados como información periférica relacionada con la recepción de la noticia y que directamente no se preguntaron (descripción de la ropa que se llevaba puesta, estado del clima); (4) expresiones o pensamientos específicosque vinieron a la mente justo cuando se escuchó la noticia (v.g., pensé:jcasi que no!= 1; pensé en todo el dolor que había causado $=0$ ). Los dos criterios adicionales para calificar los detalles entregados por los participantes fueron propuestos tomando en cuenta que los criterios de Finkenaueret al., se circunscriben a aspectos espaciales, mientras que hay detalles de pensamiento o personales que podrían indicar también que se formó o no el MFB. El puntaje máximo que se puede alcanzar en este apartado es 5.

Memoria del evento (ME). Los participantes respondieron (a) fecha exacta de la muerte de Osama bin Laden, (b) día de la semana, (c) hora de la muerte, (d) número de personas que murieron con él, (e) nombre del lugar donde murió (país y/o ciudad) y (f) qué sucedió con el cadáver. La prueba original descrita por Luminet et al., (2004) cuenta con nueve preguntas acerca de la ME de los ataques del 11-S. Sin embargo, cabe recordar que ese hecho en específico estaba cargado de información e implicó un ataque simultáneo al Pentágono. Para el presente acontecimiento, se buscó que las preguntas siguieran las características del cuestionario original, recogiendo los hechos más relevantes. En cuanto a la calificación, las respuestas fueron valoradas de 0 a 2 , siendo $0=$ respuesta incorrecta; $1=$ respuesta parcialmente correcta y $2=$ respuesta correcta. Para las respuestas a las preguntas a, b y cse tomó en cuenta que la muerte de bin Laden, conforme a medios internacionales, se registró el lunes 2 de mayo del 2011 entre las 7:20 y las 8:00 a.m., hora pakistaní. Dicho horario corresponde en Colombia al domingo 1 de mayo entre las 9:20 y las 10:00 p.m. Las respuestas, por tanto, debieron calificarse de acuerdo con estas dos informaciones. Se asumió que no podía penalizarse ninguna de las dos fechas como parcialmente correcta. Por lo tanto, a cualquiera de las dos se le otorgaron 2 puntos. Si el participante contestaba que la muerte fue el 30 de abril o el 3 de mayo, se le daba un (1) punto. Cualquier otra respuesta fue puntuada con 0 . Este mismo criterio se siguió para la calificación acerca del día dela muerte: domingo o lunes fueron puntuados con 2 puntos, mientras que respuestas como sábado o martes, recibieron 1 punto. En el caso de la hora de la muerte, los 2 puntos se le otorgaron a respuestas que estuviesen entre las dos horas antes o después del evento (v.g., 11:15 p.m. del domingo; 6:00 a.m., del lunes, hora de Pakistán), un punto a reportes de 3 horas antes o después del hecho y 0 puntos a cualquier otra hora diferente o a reportes del momento del día (v.g., en la madrugada $=0$ ).

Para la pregunta $d$, el reporte oficial indica que cuatro personas más fueron asesinadas junto 
con bin Laden, sin embargo, esta información, no es susceptible de constatación. Para objeto de la calificación del cuestionario, se entregaron dos puntos a los participantes que dieran el número exacto de personas muertas reportadas por los medios y un punto para respuestas entre 2 y 6 asesinados. Respuestas diferentes se calificaron con 0. La pregunta acerca del lugar dónde se registró la muerte se calificó tomando en cuenta la confusión inicial. Algunos medios informaron que el operativo se había llevado a cabo en Islamabad. Luego se informó que se registró en un lugar cercano a esa ciudad. Finalmente, los medios confirmaron que el operativo tuvo lugar en Abbottabad. Por esa razón a los participantes se les pidió que dijesen el país o la ciudad para obtener un punto. En este caso, la ciudad de Islamabad fue tomada como correcta, puesto que los reportes iniciales de los noticieros así lo divulgaron. Si el participante recordaba tanto la ciudad como el país obtenía 2 puntos. En último lugar, las respuestas a la pregunta $\mathrm{f}$ se puntuaron dando valor de 1 punto si el participante mencionaba que fue lanzado al mar y dos puntos si complementaba la respuesta con alguna mención al rito que las autoridades americanas dijeron que se realizó antes de arrojarlo en un punto indeterminado del océano. De la anterior descripción puede deducirse que para la calificación de un instrumento de este tipose toman en cuenta muchos detalles, así que la documentación y seguimiento de los hechos debe ser estrecho para evitar la inclusión de datos anteriores $\mathrm{o}$ posteriores al momento de la medición.

Evaluación de la novedad y la sorpresa. A los participantes se les preguntó (a) qué tan común y (b) qué tan significativo fue el evento para ellos. Además se les preguntó (c) qué tan sorprendente y (d) qué tan esperable fue la muerte de Osama bin Laden. Los ítems estaban organizados en escala de 1 "nada" a 7 "mucho". Los puntajes del ítem (a) y (d) fueron invertidos.

Evaluación de la importancia/consecuencia. En una escala de 1 a 7 , siendo 1 "nada" y 7 "mucho", los participantes calificaron qué importancia tuvo la muerte de Osama bin Laden (a) internacionalmente, (b) para el país, (c) para su familia y amigos, (d) para usted. En la misma escala, los participantes también contestaron en qué medida la muerte de Osama bin Laden tuvo consecuencias para ellos.

Intensidad de emociones asociadas al evento. Los participantes cuantificaron de 1 a 7 , siendo 1 "nada" y 7 "mucho", (a) qué tanto lo alteró la noticia de la muerte, (b) qué tan conmovido se sintió, (c) qué tanto le impactó.

Actitudes. Las actitudes se midieron a través de cuatro preguntas, dos de ellas traducidas del cuestionario original y dos más creadas ad hoc buscando que se adecuaran al evento determinante de la MFB. De tal modo, los participantes mensuraron en una escala de 1 a 7 (a) qué tanto les agradan los Estados Unidos de América como país, (b) qué tanto les agradan los ciudadanos de los Estados Unidos, (c) qué tanto apoyan la búsqueda de un fugitivo en un país extranjero por parte del país afectado y, finalmente, (d) qué tanto apoyan el asesinato de fugitivos en países extranjeros por parte del país afectado.

Repetición. Los participantes midieron de 1 a 5, siendo 1 "ninguna" y 5 "quince o más veces", (a) con qué frecuencia tuvieron pensamientos, recuerdos o imágenes relacionadas con la muerte de bin Laden en los últimos tres (3) días, (b) cuántas veces hablaron de la muerte de Osama en los últimos 3 días y (c) en los últimos 3 días, cuántas veces siguieron el caso en periódicos o noticieros.

El punto de referencia de los últimos 3 días, se asumió siguiendo la recomendación de Luminet y Curci (2009) para evitar el efecto techo en los análisis.

Conocimiento previo. En esta dimensión se incluyeron las siguientes preguntas (a) Nacionalidad de Osama bin Laden, (b) en qué país se sospechaba que estaba escondido, (c) por qué razón era conocido a nivel mundial (d) nombre del grupo terrorista que lideraba.

Para la pregunta (a) las respuestas fueron calificadas como 0 y 1 , aceptando como correctas respuestas del tipo Árabe, Saudí o de Arabia Saudita. Las respuestas a las preguntas (b, c, d) se calificaron de 0 a 3 . Referente a la pregunta (b), 
aunque el sitio en el cual bin Laden se ocultaba no estuvo claro hasta el desenlace del operativo, las agencias de inteligencia norteamericanas suponían que se ocultaba en algún lugar de Pakistán. Por ello, las respuestas que mencionaran dicho país obtuvieron 2 puntos. Respuestas donde se mencionase a Afganistán como posible escondite recibieron 1 punto, dado que los Estados Unidos suponían que los grupos Talibanes le ofrecían refugio. Respuestas que brindasen información adicional recibieron un punto extra (v.g., en Pakistán cerca de la frontera con Afganistán, al noreste de Pakistán).

Las preguntas restantes se calificaron de 0 a 3 , obteniendo el puntaje máximo respuestas que incluían información complementaria. Por ejemplo, para la pregunta (c) una respuesta escueta que apuntaba a que era conocido por terrorista, obtenía 1 punto; si a la respuesta se le adicionaba que fue el responsable de los ataques a las torres gemelas o que era un millonario Saudí aliado de los EE.UU en tiempos de la guerra fría, se le asignaba un punto extra.

\section{Procedimiento}

Los participantes leyeron y firmaron un consentimiento informado en el cuál se les resumían los aspectos centrales de la investigación, el tratamiento que se haría con los datos recogidos y la posibilidad que tenían de retirarse en cualquier etapa del proceso. Posteriormente, rellenaron el instrumento de manera individual. La aplicación completa tomó 30 minutos en promedio y se desarrolló entre los 4 y 19 días posteriores al evento.

\section{Análisis de datos}

Como la asunción de base es que cada grupo de ítems representa una dimensión dentro de la configuración de un Recuerdo Flashbulb, se realizaron análisis individuales por dimensión. A esta solución se le conoce como "forma canónica" (Ferrando \& Anguiano-Carrasco, 2010). Siguiendo las recomendación de Ferrando y Lorenzo-Seva (2014) de analizar las características de la prueba para tomar la mejor decisión en cuanto a la solución factorial a elegir, se realizaron análisis sobre matrices policóricas en un primer momento y de Pearson en un segundo momento, cada una de ellas con simulaciones de 500 matrices aleatorias. La extracción de una única dimensión, en cada caso, se determinó a través del Análisis Paralelo (AP) (Timmerman\& Lorenzo-Seva, 2011). Finalmente, el Análisis Factorial (AF) se realizó mediante el método de Análisis Factorial de Rango Mínimo (MRFA), método que permite discriminar la varianza común del total de la varianza explicada.

Las extracciones, tanto en las matrices de Pearson como en las policóricas, advirtieron un único factor que explicaba la mayor cantidad de varianza común explicada para cada dimensión. Al analizar las características de la prueba y contrastar las cargas factoriales, se puso de manifiesto que, tal $\mathrm{y}$ como ha señalado Dominguez-Lara (2014), las soluciones obtenidas sobre la matriz de correlaciones policóricas eran más ajustadas. Además, este tipo de análisis se muestra idóneo para el análisis de pruebas en el campo social (Holgado, Sánchez, \& Navas, 2011). Por esa razón se descartaron las soluciones obtenidas sobre las matrices de correlación de Pearson.

Se calcularon la media y la desviación estándar para cada ítem, las cargas factoriales y la consistencia interna de lasdimensionesa través del coeficiente alfa de Cronbach, además se calculó el intervalo de confianza para dicho coeficiente.Para el análisis de los datos se utilizaron los paquetes estadísticos SPSS 21 y FACTOR (Lorenzo-Seva $\&$ Ferrando, 2013).

\section{Resultados}

En las medias reportadas en la Tabla 2, puede observarse que la mayoría de personas tenían recuerdos específicos del momento de la recepción de la noticia de la muerte de bin Laden, además, consideraron el acontecimiento como significativo y novedoso pero esperable, con bajos niveles de consecuencialidad y relevante tanto para Colombia como internacionalmente. En general, los participantes recordaron el número de personas que murieron en el operativo y el destino del cuerpo sin vida de bin Laden. En cuanto a las actitudes, las personas mostraron agrado por los Estados Unidos y sus ciudadanos. También apoyaron la búsqueda de fugitivos por fuera de 
Tabla 2. Análisis factorial para cada una de las dimensiones de la prueba de MFB de la muerte de Osama bin Laden realizado sobre una matriz de correlaciones policóricas

\begin{tabular}{|c|c|c|c|c|c|}
\hline Dimensión & EV & $\begin{array}{l}\text { Varianza } \\
\text { común } \\
\text { explicada }\end{array}$ & $\begin{array}{l}\text { Carga } \\
\text { factorial }\end{array}$ & $\alpha[\mathrm{IC} 95 \%]$ & $M(D T)$ \\
\hline $\begin{array}{l}\text { MFB Muerte de } \\
\text { OBL }\end{array}$ & 2.458 & $78.37 \%$ & & $.792[.751-.827]$ & \\
\hline Ítem a & & & .655 & & $.93(.26)$ \\
\hline Ítem b & & & .879 & & $.95(.22)$ \\
\hline Ítem c & & & .457 & & $.96(.19)$ \\
\hline Ítem d & & & .786 & & $.92(.27)$ \\
\hline Ítem e & & & .656 & & $.94(.24)$ \\
\hline ME & 2.248 & $70.68 \%$ & & $.69[.633-.739]$ & \\
\hline Ítem a & & & .948 & & $.003(.17)$ \\
\hline Ítem b & & & .941 & & $.96(.96)$ \\
\hline Ítem c & & & .400 & & $.24(.64)$ \\
\hline Ítem d & & & .300 & & $.66(.71)$ \\
\hline Ítem e & & & .325 & & $.50(.54)$ \\
\hline Ítem $\mathrm{f}$ & & & .330 & & $.74(.50)$ \\
\hline Nove/Sor & 1.761 & $75.19 \%$ & & $.648[.586-.703]$ & \\
\hline Ítem a & & & .360 & & $3,37(2.41)$ \\
\hline Ítem b & & & -.803 & & $5.87(1.81)$ \\
\hline Ítem c & & & -.955 & & $5.69(3.79)$ \\
\hline Ítem d & & & .274 & & $4.21(2.32)$ \\
\hline Impor/Conse & 2.324 & $74.72 \%$ & & $.734[.684-.777]$ & \\
\hline Ítem a & & & .207 & & $6.56(1.04)$ \\
\hline Ítem b & & & .678 & & $4.22(1.99)$ \\
\hline Ítem c & & & .779 & & $3.09(2.22)$ \\
\hline Ítem d & & & .943 & & $3.06(2.28)$ \\
\hline Ítem e & & & .571 & & $1.90(1.73)$ \\
\hline Emociones & 1.556 & $99 \%$ & & $.730[.679-.774]$ & \\
\hline Ítem a & & & .763 & & $1.86(1.66)$ \\
\hline Ítem b & & & .869 & & $2.04(1.72)$ \\
\hline Ítem c & & & .467 & & $3.94(2.31)$ \\
\hline Actitudes & 2.301 & $75.06 \%$ & & $.804[.765-.837]$ & \\
\hline Ítem a & & & .818 & & $4.39(2.12)$ \\
\hline Ítem b & & & .704 & & $4.33(1.94)$ \\
\hline Ítem c & & & .811 & & $4.82(2.24)$ \\
\hline Ítem d & & & .692 & & $4.11(2.36)$ \\
\hline Repetición & 1.724 & $100 \%$ & & $.797[.757-.831]$ & \\
\hline Ítem a & & & .677 & & $1.88(1.18)$ \\
\hline Ítem b & & & .850 & & $2.14(1.22)$ \\
\hline Ítem c & & & .737 & & $2.09(1.28)$ \\
\hline Conoc/Prev & 1.422 & $89.86 \%$ & & $.639[.575-.695]$ & \\
\hline Ítem a & & & .677 & & $.25(.48)$ \\
\hline Ítem b & & & .435 & & $.82(.88)$ \\
\hline Ítem c & & & .399 & & $.92(.55)$ \\
\hline Ítem d & & & .784 & & $.77(.45)$ \\
\hline
\end{tabular}

$\mathrm{MFB}=$ Recuerdo Flashbulb; OBL= Osama bin Laden; ME= Memoria del evento; Nove/Sor= Evaluación de Novedad/ Sorpresa; Impor/Conse= Evaluación Importancia/Consecuencialidad; Conoc/Prev= Conocimiento previo; EV= Autovalor; VCE= Varianza Común Explicada; $\alpha=a l f a$ de Cronbach; M= Media; DT= Desviación típica. 
las fronteras y su asesinato.

Las cargas factoriales de los ítems son aceptables excepto en el ítem d de la dimensión Novedad/Sorpresa y en él ítem a de la dimensión Importancia consecuencia.

Los coeficientes de confiabilidad se ubicaron en un rango de .639- .804. Valores aceptables que indican una buena consistencia interna de las dimensiones; no obstante al realizar el cálculo de los intervalos de confianza, dos de ellas mostraron límites inferiores bajos: Novedad/Sorpresa [.586 - .703] y Conocimiento previo [.575 - .695].

\section{Discusión}

El objetivo principal del presente estudio fue establecer las propiedades psicométricas de un instrumento adaptado para la investigación de MFB. Estudios relacionados con este tipo de recuerdos autobiográficos presentan la particularidad de que las pruebas deben adaptarse muy rápidamente (en algunos casos, horas) al acontecimiento que se desea evaluar y los criterios para su calificación posterior deben estudiarse con detenimiento. Dado el actual interés en los aspectos sociales relacionados con la formación de MFB, y con investigaciones que involucran el comportamiento de los consumidores (Roehm, 2016), es importante poner a disposición de los investigadores iberoamericanos una prueba modelo.

La prueba evaluada en este artículo responde en su totalidad a una conceptualización teórica en torno a los mecanismos que subyacen a la formación de un MFB. En la investigación realizada por Brown y Kulik (1977) se plantearon no solo los componentes del constructo MFB, si no también un modelo básico que intentó explicar cuáles serían las dimensiones y las relaciones entre estas, que ayudarían a comprender las particularidades de los MFB. Desde esa primera investigación hasta la actualidad se han propuesto nuevos modelos e incluido nuevas dimensiones. No obstante, Luminet (2009) mostró que hay cuatro dimensiones constantes: la reacción de sorpresa, la evaluación cognitiva de la importancia del evento, la repetición abierta o encubierta y la activación emocional. En cualquier caso, un aspecto central es que cada componente de la prueba pueda considerarse como una dimensión aislada. En el presente instrumento esta condición se guarda. Las extracciones de factores realizadas sobre matrices policóricas muestran en todos los casos un único factor que explica la mayor parte de la varianza común.

En cuanto a las estructuras internas de cada dimensión se observa que las cargas factoriales son satisfactorias, aunque dos ítems mostraron valores bajos: ítem d (Qué tan esperable fue el evento) de la dimensión Novedad/Sorpresa y el ítem a (Qué importancia tuvo la muerte de Osama bin Laden internacionalmente) de la dimensión Importancia/Consecuencia. No obstante, por criterios teóricos dichos ítems se conservaron en cada dimensión. Una explicación preliminar para valores tan bajos podría relacionarse con el tipo de evento y población evaluada. En efecto, la muerte de bin Laden, no necesariamente desencadenó respuestas altas de sorpresa, puesto que constantemente en medios de comunicación se presentaban noticias que hablaban de la persecución a la que era sometido, por tanto, la noticia de su muerte era esperable. Estudios acerca de eventos poco sorpresivos, pero de impacto mundial han sido estudiados (Tinti, Schmidt, Sotgiu, Testa, \& Curci, 2009) mostrando que en términos generales responden a los presupuestos básicos de formación de MFB. Curci, Luminet, Finkenauer, \& Gilse (2001) fueron los primeros en reportar MFB de hechos esperables y poco novedosos. En su caso fue la muerte del expresidente Miterrand.

Dado que este fenómeno contradecía la teoría, Curci y Luminet (2009) analizaron de nuevo los datos de esta investigación, asumiendo que hechos esperables podrían generar MFB, y su formación podría ser explicada a través del modelo propuesto por Finkenauer et al., (1998). El mencionado modelo asume dos vías para la formación de una MFB: por un lado, una vía directa relacionada con el impacto emocional representado por la novedad y la sorpresa generada por el evento. De otro lado, habría un camino indirecto, y el predictor final de la formación del MFB sería la memoria del evento como tal. Efectivamente, esto autores encontraron que la última vía ayudaba a comprender la formación de MFB para hechos esperables, es 
decir, aquellos con bajo impacto emocional. Esto quiere decirque, a pesar del bajo impacto emocional, es posible estudiar recuerdos de noticias de trascendencia internacional más allá de la valoración a priori que pueda hacerse de su impacto.

En cuanto a la consistencia de la prueba, el rango de valores del coeficiente alfa de Cronbachfue aceptable. Dominguez-Lara y Merino-Soto (2015) han recomendado la inclusión en los reportes de confiabilidad del intervalo de confianza para mostrar los valores entre los cuáles se encontraría el valor verdadero del alfa en la población. En el presente estudio las dimensiones que presentaron el límite inferior más bajo en el intervalo de confianza en el índice de confiabilidad fueron Novedad/Sorpresa y Conocimiento previo. En esta última, cabe considerar el hecho de que la dimensión es bastante amplia y solo se eligen un número reducido de ítems que pudiesen representar los principales sucesos del evento de manera precisa. No obstante, para hechos sociales complejos, cargados de información y con tendencia a la acumulación de nueva información con el paso de las horas y los días, las preguntas pueden perder especificidad y validez. Se necesitaría para futuros cuestionarios ampliar el número de preguntas para tener una mayor representación del evento.

En los valores reportados para el instrumento de Curci y Luminet (2006), la confiabilidad más baja fue reportada para la dimensión de especificidad de MFB con un alfa de .573 seguido de Importancia/consecuencialidad con .699. En dicho instrumento, ningún alfa alcanzo un nivel de .80 , no obstante los autores consideraron que los valores eran aceptables.

En cualquier caso, es necesario refinar más los procesos de creación de cada dimensión, intentando precisar las variables observables, teniendo en cuenta que en muchos casos los niveles de confiabilidad no serán tan altos, pues la prueba piloto o no podrá llevarse a cabo o se hará muy rápidamente antes de la aplicación final del instrumento.

La prueba presentada es el ejemplo de una prueba particular: parte de ella debe ser elaborada de acuerdo con el evento que se desee estudiar; por esta razón es importante que los investigadores interesados en este campo revisen muy bien las técnicas de calificación y de análisis que deben utilizar para alcanzar validez en sus estudios.

En la actualidad hay un renovado interés por los estudios de memoria en los cuales se ven involucrados hechos sociales. Particularmente en Colombia, donde se acaba de firmar un proceso de paz con el grupo guerrillero más longevo del continente, muchas voces se levantan haciendo énfasis en la necesidad de recordar como modo de resarcimiento para las víctimas del conflicto. Las características de las MFB son propicias para el estudio de parte de la historia de este conflicto desde las voces de los diferentes actores. Así mismo, microestudios en este campo ayudarían a entender las relaciones entre la memoria de hechos sociales y traumas posconflicto. Por último, la crueldad y extensión temporal del conflicto colombiano logró que muchos hechos luctuosos (tomas guerrilleras, atentados con carros cargados con explosivos, destrucción de pueblos enteros) fuesen normalizados $\mathrm{y}$ por tanto careciesen de MFB (Tamayo-Agudelo, 2011). Sin embargo, muchos otros hechos relacionados con la historia reciente de Colombia son susceptibles de generar MFB. Uno de ellos podría ser la firma del tratado de paz con el grupo armado FARC, que da fin al conflicto armado más antiguo de América, o también, los logros deportivos en competiciones internacionales. Vale la pena, entonces, explorar las MFB del pueblo colombiano como un modo posible de establecer un corpus de recuerdos asociados a hechos sociales.

Para finalizar es necesario dejar claro que la principal limitación de este estudio es el hecho de que no se hubiese realizado una segunda aplicación de la prueba, buscando evaluar la consistencia de las MFB, y de manera secundaria, la confiabilidad test-retest del instrumento. Una segunda falencia es la no aleatoriedad de la muestra, hecho este bastante común en estudios no experimentales de memoria. Para futuras investigaciones, quizás sea más fácil realizar pruebas retest y sería ideal realizar rápidos muestreos probabilísticos para evaluar poblaciones luego de que acontezca algún suceso inesperado. 


\section{Referencias}

Brown, R., \& Kulik, J. (1977). Flashbulb memories. Cognition, 5, 73-99. doi: http://dx.doi.org/10.1016/00100277(77)90018-X

Conway, M., Anderson, S. J., Larsen, S. F., Donnelly, C. M., McDaniel. M. A., McClelland, A. G. R., Rawles, R. E., \& Logie, R. H. (1994). The formation of flashbulb memories. Memory \& Cognition, 22, 326-343.

Curci, A., \& Luminet, O. (2006). Follow-up of a cross-national comparison on flashbulb and event memory for the September 11th attacks. Memory, 14, 329-344. doi: 10.1080/09658210 500340816

Curci, A., \& Luminet, O. (2009). Flashbulb memories for expected events: A Test of the Emotional-Integrative Model. Applied Cognitive Psychology, 23, 98-114. doi: 10.1002/acp.1444

Curci, A., Luminet, O., Finkenauer, C., \& Gisle, L. (2001). Flashbulb memories in social groups: A comparative test-retest study of the memory of French President Mitterrand's death in a French and Belgian group. Memory, 9, 81-101. doi: 10.1080/09658210042000120

Dominguez-Lara, S. (2014). ¿Matrices Policóricas/Tetracóricas o Matrices Pearson? Un estudio metodológico. Revista Argentina de Ciencias del Comportamiento, 6(1), 39-48.

Dominguez-Lara, S., \& Merino-Soto, C. (2015). ¿Por qué es importante reportar los intervalos de confianza del coeficiente alfa de Cronbach? Revista Latinoamericana de Ciencias Sociales, Niñez y Juventud, 13(2), 1326-1328.

Er, N. (2003). A New Flashbulb Memory Model Applied to the Marmara earthquake Applied Cognitive Psychology 17, 503-517. doi: 10.1002/acp.870

Finkenauer, C., Luminet, O., Gisle, L., ElAhmadi, A., Van der Linden, M., \& Philippot, P. (1998). Flashbulb memories and the underlying mechanisms of their formation: Toward an emotional-integrative model. Memory \& Cognition, 26, 516-531.doi: 10.3758/BF03201160

Fridja, N., Kuipers, P., \& terSchure, E. (1989). Relation among emotion, appraisal end emotion al action readiness. Journal of personality and social psychology, 57(2), 212228.doi: 10.1037/0022-3514.57.2.212

Ferrando, P. J., \& Anguiano-Carrasco, C. (2010). El análisis factorial como técnica de investigación en psicología. Papeles del Psicólogo, 31(1), 18-33.

Ferrando, P. J., \& Lorenzo-Seva, U. (2014). El análisis factorial exploratorio de los ítems: Algunas consideraciones adicionales. Anales de Psicología, 30, 1170-1175. doi: 10.6018/analesps.30.3.199991

Holgado, F., Sánchez, A., \& Navas L. (2011). Análisis de la estructura de la Escala de Actitudes hacia la Inmigración en una muestra de estudiantes chilenos. Revista Iberoamericana de Diagnóstico y Evaluación - e Avaliação Psicológica, 1(29), 97-113.

Livingston, R. (1967). Reinforcement. In G. Quarton, T. Melenchunk, \& F. Schmitt (Eds.).The neurosciences: A study program. New York: Rockfeller University Press.

Lorenzo-Seva, U., \& Ferrando, P. J. (2013). FACTOR 9.2. a comprehensive program for fitting exploratory and semiconfirmatory factor analysis and IRT Models. Applied Psychological Measurement, 37, 497-498. doi: $10.1177 / 0146621613487794$

Luminet, O. (2009). Models for the formation of flashbulb memories. En O. Luminet \& A. Curci. (Eds). Flashbulb memories. New Issues and New Perspectives. (pp. 51-73). New York: Psychology Press.

Luminet, O., \& Curci, A. (2009). The 9/11 attacks inside and outside the US: Testing four models of flashbulb memory formation across groups and the specific effects of social identity. Memory, 17, 742-759 doi: 10.1080/09658210903081827

Luminet, O., Curci, A., Marsh, E., Wessel, I., Constantin, T., Gencoz, F., \& Masao, Y. (2004). The Cognitive, Emotional and Social Impacts of the September 11 Attacks: Group differences in memory for the reception context and the determinants of Flashbulb Memory. The Journal of General Psychology, 131, 197-224.doi: 10.3200/GENP.131.3.197224

Montero, I., \& León, O. G. (2007). A guide for naming research studies in Psychology. 
International journal of Clinical and Health Psychology, 7, 847-862.

Moreira, J. M., \& Gamboa, P. (2016). Inventário de Estados Afetivos-Reduzido: Uma medida multidimensional breve de indicadores emocionais de ajustamento. Revista Iberoamericana de Diagnóstico y Evaluación - e Avaliação Psicológica, 1(41), 132-144.

Roehm, M. L. (2016). An exploration of flashbulb memory. Journal of Consumer Psychology, 26(1), 1-16. doi: http://dx.doi.org/10.1016/j.jc ps.2015.06.004

Tamayo-Agudelo, W. (2011). Introducción a las Memorias Flashbulb (MFB). Pensando Psicología, 7(12), 119-126. Disponible en: goo.gl/WOdow8

Timmerman, M. E., \& Lorenzo-Seva, U. (2011). Dimensionality assessment of ordered polytomous items with parallel analysis. Psychological Methods, 16, 209-220. doi: 10.1037/a0023353

Tinti, C, Schmidt, S., Sotgiu, I., Testa, S., \& Curci, A. (2009). The role of importance/consequentiality appraisal in Flashbulb memory formation: The case of the death of Pope John Paul II. Applied Cognitive Psychology, 23, 236-253. doi: 10.1002/acp.1452 